\title{
The Barium Carbonate Procedure for the Preparation of Glass Capillary Columns; Further Informations and Developments
}

\author{
K. Grob / G. Grob / K. Grob Jr. \\ GC-Laboratory, ETH Zürich, EAWAG, CH-8600 Dübendorf, Switzerland
}

\section{Summary}

The present second report on the preparation of glass capillary columns, based on producing a crystal layer of barium carbonate on the internal capillary surface, completes the information given in the first report mainly in three directions. First, more practical experience with long term use is presented, showing this column type to be very promising because of universality, durability, simplicity, and efficiency. Second, more insight is given into the underlying processes, as well as into the produced effects. A new aspect concerns the double function of the barium carbonate layer, namely roughening the internal column wall and covering the catalytically active silica surface. Third, more elaborate procedures reducing the particle size of barium carbonate crystals are discussed. On finer particles more homogeneous films, yielding higher separation efficiency, are obtained. Detailed practical directions to new procedures are given.

\section{Practical Experiences with the Basic Procedure}

The new procedure, as described in our first report [1], has been applied in several laboratories, working with different background, training of personel, and equipment. This application has shown the procedure to have important merits, part of which we were hoping for, while others became evident only during application. These merits essentially are

- Quick success in nonspecialized laboratories;

- Higher reproducibility than with any other procedure;

- Independence on glass variety;

- Increased thermal stability of coatings as a result of decreased catalytic activity of the barium carbonate surface;

- Application for surface roughening as well as for smooth surface covering;

- Insensitivity to water;

- Suitability for subsequent modification producing specific characteristics.
In our first report we emphasized the aims of simplicity, reproducibility, and stability at the possible expense of top separation efficiency of polar columns (the efficiency of apolar coatings does not strongly depend on details of surface pretreatment). In the same report we mentioned that, with additional care, some characteristics could be improved. It is the purpose of the present paper to provide the necessary knowledge, as well as various additional information on the procedure.

\section{Deeper Insight into the Procedure}

\subsection{The Crystallization Process}

The principle of the procedure is the production of barium carbonate crystals growing on a glass surface wetted with barium hydroxide solution, upon contact with carbon dioxide. Under appropriate conditions all crystals start growing on the solid surface, rather than in the free liquid phase, thus producing a crystal layer firmly adhering to the glass. This experimental fact has not yet been understood theoretically. Our present interpretation that barium ions complexed on the silica surface are more powerful crystallization nuclei than hydrated ions tries to describe the observed reaction, rather than the underlying mechanism (Fig. 1). It is not easy to understand how carbonate ions may pass the water layer down to the silica surface without reacting with the amply (up to $6 \% \mathrm{Ba}(\mathrm{OH})_{2}$ ) occuring barium ions in the free liquid phase. The process is perfectly reproducible, provided the following conditions, governing complexation of barium ions, are fulfilled: Sufficiently "opened" silica structure offering silanol groups, $\mathrm{pH}$ of water layer not lower than 10 , no dissolved substances competing with barium ions for complexing sites.

\subsection{The role of Glass Variety}

Under the above-mentioned conditions barium carbonate crystals grow on any kind of glass. On the other hand, the glass structure influences size, shape, and distribution of the crystals. On untreated soft glass, for instance, smaller and less distant particles are formed than on untreated Pyrex glass. However, these differences become the smaller, the more efficiently the glass has been subjected to acidic leaching. We interprete this observation by assuming that acidic leaching eliminates components such as metal ions, boron complexes etc., leaving, when carried out thoroughly, 


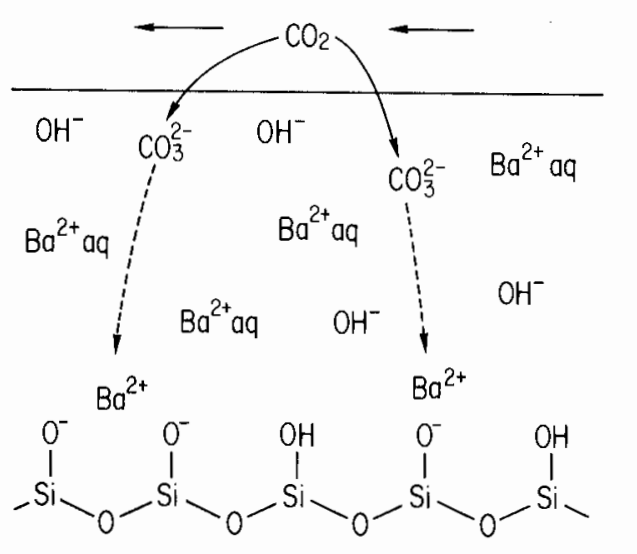

moving

$\mathrm{CO}_{2}$ gas

Fig. 1

Schematically presented conditions of the crystallization process. The question remains open: how do carbonate ions diffuse through the approx. $1 \mu \mathrm{m}$ thick water film without reacting with hydrated barium ions?

an essentially pure silica surface. Thus, with increasing intensity of leaching, the surfaces of different glasses become more similar.

One of the leading arguments in the selection of glass type has long been acidity. From soft glass basic columns were produced; we were obliged to use borosilicate glass when neutral or acidic columns were wanted. The latter fact was accepted reluctantly since borosilicate glasses were known to exhibit much stronger adsorption effects than soft glasses. It seams that, with proper leaching, these differences almost disappear. Thus, acidity is no longer a fixed precondition of glass type, but can be preselected by correspondingly building up the appropriate interlayer on the silica surface. For the moment, even after leaching 20 hours at $150^{\circ}$ with $20 \% \mathrm{HCl}$, we still observe a small difference in adsorption activity between soft and borosilicate glass.

\subsection{Producing Finer Barium Carbonate Particles}

Polar columns prepared according to the basic procedure as described in our first report show relatively modest separation efficiences. The reason obviously is that the film-supporting particles are excessively large and distant. Increasing the separation efficiency thus means producing finer and less distant particles. A known measure to accomplish this is adding polyols to the barium hydroxide solution. Our trials in this field were not encouraging, since barium carbonate particles moving in the free water phase were observed.

Another measure to reduce particle size is lowering the crystallization temperature. At first we had, again, poor results. The particles were formed on the solid surface exclusively. However, they adhered less to the surface. Part of them were displaced when a plug of acetone was pushed through the capillary to wash out the remaining water. This never occurred after crystallization at $80^{\circ}$, as reported earlier.

We then discovered the simple reason of the trouble. At room temperature much more carbon dioxide is dissolved in water than at $80^{\circ}$. This leads to some shift from the originally formed $\mathrm{BaCO}_{3}$ to the more soluble $\mathrm{Ba}\left(\mathrm{HCO}_{3}\right)_{2}$. Appearently this shift affects the area where the particles adhere to the glass, thus reducing their bonding to the solid surface. The problem is perfectly overcome by including two additonal treatment steps (steps $C$ and D in Fig. 2) before the water is eliminated. Step C, flushing with air or nitrogen, reduces the carbon dioxide concentration in water sufficiently to keep the particles in position when the subsequent heating (step D) causes the water film to break up into drops. The combined effect of heating and flushing (D) finally assures perfect stability of the barium carbonate layer.

An absolute precondition for the functioning of this treatment is an unbroken water film as produced by the moving $\mathrm{Ba}(\mathrm{OH})_{2}$ plug and remaining up to and including step C.Premature film breaking, i.e. breaking when the

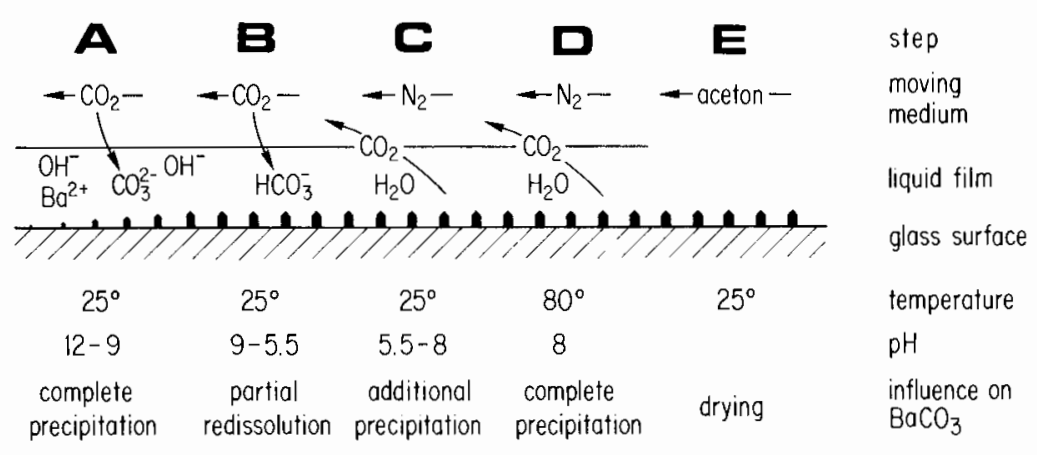

Fig. 2

- Schematic presentation of the consecutive steps making up crystallization at room temperature, including their specific conditions. The critical step is $\mathrm{B}$, during which the crystals are less firmly bound to the glass surface. Thus the water film must remain homogeneous as long as $\mathrm{CO}_{2}$ gas flows in the column (end of step $\mathrm{C}$ ). 
$\mathrm{CO}_{2}$ concentration in water is still high, leads to moving water plugs which distroy the homogeneous barium car. bonate layer by displacing loosened particles. The neces. sary film stability requires a more efficient detergent than indicated in our first paper (details see chapter 3.2).

Crystallization at room temperature greatly reduces particle size and inter-particle distance. Also, it influences the shape of the particles. Details will be discussed in a separate paper based on scanning electron micrographs. Polar coatings deposited on a barium carbonate layer obtained at room temperature are more homogeneous, thus yielding increased separation efficiency. Besides this improvement, we prefere cold precipitation for a second and independant reason. The intense acidic leaching covers the glass with a pure silica layer. This layer is partly dissolved or displaced under the influence of strong bases at elevated temperatures. At room temperature no harm to the silica layer is observed, provided the layer has been sufficiently dehydrated (see chapter 3.1).

We wish to emphasize that the above indications in no way replace our previous instructions (crystallization at $80^{\circ}$ on untreated glass) which described a simpler and shorter procedure leading to a durable though rougher product.

\subsection{Barium Carbonate Versus Silica Surface}

The silica surface, the production and conservation of which we emphasize in the foregoing chapters, is the ideal support for a homogeneous barium carbonate layer. On the other hand we must stress that uncovered silica surface is an unsuitable support for organic coatings. To our knowledge this simple statement is missing in the literature. We deduce it from an enormous wealth of failure and disappointment gathered in our laboratory. A twofold detrimental effect exerted by a silica surface may be described.

Silica catalyses - we ignore by which mechanism - the breakdown of most organic macromolecules, e.g. all polyglycols, polyesters, aromatic polyethers, polar silicones. The simple consequence is that films of such substances deposited on a silica surface show similar bleeding and durability at $40-50^{\circ}$ lower temperatures compared with the same films on barium carbonate as a support. In other words: while we indicated upper temperature limits for Ucon HB 5100, Emulphor, and PG $20 \mathrm{M}$ to be 180,190 , and $200^{\circ}$ resp. we now find 230,240 , and $250^{\circ}$ resp. for the same phases on our barium carbonate support. At first we suspected this drastic difference to derive from a difference in acidity. We therefore produced Ucon HB columns, both with $\mathrm{BaCO}_{3}$ and silica surface, showing identical acidity. After prolonged conditioning, the silica column showed a sevenfold bleed rate as compared to the barium carbonate column checked at the same temperature. The next question was, whether the influence caused by silica was a specific one, producing specific break down products. As Fig. 3 shows, this is not the case. The back. ground mass spectra, recorded at the same temperature, show the expected difference in intensity, but essentially no difference of the fragmentation pattern. We conclude
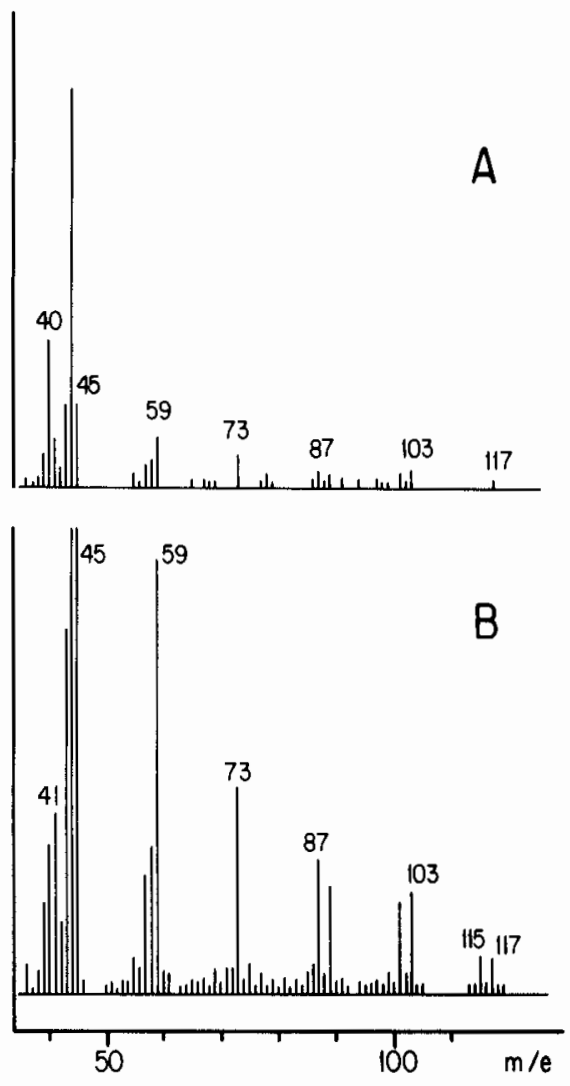

Fig. 3

- Mass spectra of column background from two neutral Ucon HB 5100 columns with identical geometry, film thickness, and obtained under identical chromatographic conditions (column temperature $200^{\circ}$ ). Column A: barium carbonate type. Column B: silica surface, produced by alcaline edging followed by acidic precipitation. Note the similar height of the argon peak 40 . Mass spectrometer Mod. 112 from VARIAN MAT, Bremen, G.E.R.

that the same break down reactions, occurring on a carbonate support, proceed, with greatly increased intensity, on a silica surface.

It is not easy to correlate our findings with results from other laboratories, since exact long term temperature limits are not often reported. A recent indirect confirmation may be deduced from the fine work by Schulte [2], as far as temperature limits for columns with silica support are indicated.

Apolar silicones are less affected by the described influence of silica. They are, however, damaged by silica in a different way. In a previous paper [3] we described a phenomenon limiting thermostability of silicone columns, namely a slow change of the film from liquid to a quasisolid state, simulating a decrease of film thickness and causing reduced retention. The experience underlying these informations were exclusively gained with silica columns! We have never observed a corresponding effect with barium carbonate columns coated with apolar silicones.

\subsection{Surface Roughening Versus Surface Covering}

An universal surface treatment has to fulfill two totally different tasks. On one hand it should perfectly cover the 
silica surface. For subsequent apolar coatings this cover should remain smooth, since unnecessary roughening would cause some loss of film homogeneity and, therefore, of separation efficiency. On the other hand, the same treatment should, by changing a fundamental parameter, produce the surface roughness, as a precondition for polar coating.

The barium carbonate treatment does fulfill both tasks, the variable parameter simply beeing the concentration of barium hydroxide. From saturated barium hydroxyde solution a rather dense crystal layer is obtained. From gradually diluted solutions the crystals become more distant, while the glass surface between the crystals still remains covered by a very thin and smooth layer of barium carbonate. When the solution is diluted $50-100$ times the crystals disappear, leaving the smooth surface cover only. It is in this concentration range also, where the layer starts beeing too weak for permanently masking the silica structure. Thus, from our present experience we recommend a 30 -fold diluted solution (ca. $0.2 \%$ Barium hydroxide) to produce the ideal basis for apolar coatings.

We must add that we have no direct proof for the existence of the above-mentioned thin cover. Our strong evidence, pointing to such a cover, is that both surfaces, treated with concentrated or with diluted solution, show the same chemical behaviour, while they do not show the characteristics of a silica surface.

\subsection{Insensitivity to Water}

The barium carbonate interlayer has been produced from an aqueous solution. It is, therefore, not affected by subsequent contact with condensed water. This fact gives rise to the question whether, and to what extent, aqueous samples may be injected onto barium carbonate columns. After broad experimental investigation we may summarize the situation as follows.

Aqueous solutions can be injected onto barium carbonate columns coated with moderately polar or polar phases exactly the same way as samples with organic solvent. The only difference is, that, with FID, no solvent peak appears. Thus, water may serve as the ideal solvent when the solvent peak has to be avoided. Even direct water injections (e.g. $2-3 \mathrm{~mm}^{3}$ with closed split valve, column temperature $70-80^{\circ}$ ) repeated over several days do not harm to such columns. Unfortunately, on the other hand, water is a poor solvent for most organic sample components, especially for the more volatile ones. For such substances water yields a poor or no solvent effect, with the result that they are not effectively trapped at column inlet, as is the case during a well functioning splitless injection. Polar sample components, for which water does show a solvent effect, normally have sufficiently low volatility to be efficiently accumulated on the column by simple cold trapping.

Water injections onto apolar columns are not recommended. While their separation efficiency is not affected by repeated water injections, a slow increase of adsorption activity is observed.
Instead of a selection of pleasant examples we prefere to present the solution of a rather hard problem, namely the analysis of liquors. Official chemists wish to estimate the series of low molecular weight alcohols and, besides, the less volatile constituents causing the flavour. Since the latter are trace substances, direct analysis involves injection of large amounts of water. For the interpretation of Fig. 4 the following details should be noticed. $0.3 \mathrm{~mm}^{3}$ were introduced onto the column at room temperature. Under these conditions small droplets of condensed water become visible in the first meter of the column. Immediately after the heavy overloading with lower alcohols almost full sensitivity is used to record the flavor section, whereby no sign of the foregoing hard treatment becomes appearent. The temperature is programmed up to $170^{\circ}$, while the commonly accepted temperature limit for Carbowax 400 is around $140^{\circ}$. This exemplifies the exceptional stability of the column, caused by the low catalytic activity of the barium carbonate surface.

\subsection{Modification of Barium Carbonate}

Our present results show the feasibility of replacing the carbonate ion in the barium carbonate surface, in situ, by other ions such as sulfate, phosphate, organophosphates. This allows the production of stable columns with intensely modified characteristics, especially with respect to acidity and adsorption. A separate paper dealing with such modifications is in preparation.

\subsection{Comparison With Other Surface Treatments}

While not attempting to deal with the complete spectrum of presently known treatments we wish to correlate the new procedure to the most common or most promising ones. In our view the only procedure offering a high degree of universality is the so-called sodium chloride procedure, i.e. treatment of soft glass with dry hydrogen chloride at elevated temperature. This treatment is at present time under intense development $[4,5]$. Thus it may well be that its lack of reproducibility it showed during the last years may be overcome. We doubt, however, whether it will become equivalent to the barium carbonate procedure in the following two respects. Firstly we wonder whether it is feasible to produce a coherent sodium chloride cover onto the silica surface without roughening the surface at the same time. Secondly it seems difficult to see how the sodium chloride surface could be modified in specific ways, e.g. to produce acidic columns.

Very valuable alternatives to our procedure are available in case specific effects rather than universality are wanted. It seems very probable, for instance, that simple and more effective procedures for the deactivation of the smooth glass surface will be worked out as a basis for improved apolar columns. These procedures will, however, not be suitable to prepare polar columns.

A further group of promising procedures starts from the principle to introduce surface-covering and surfaceroughening material from outside, rather than producing 


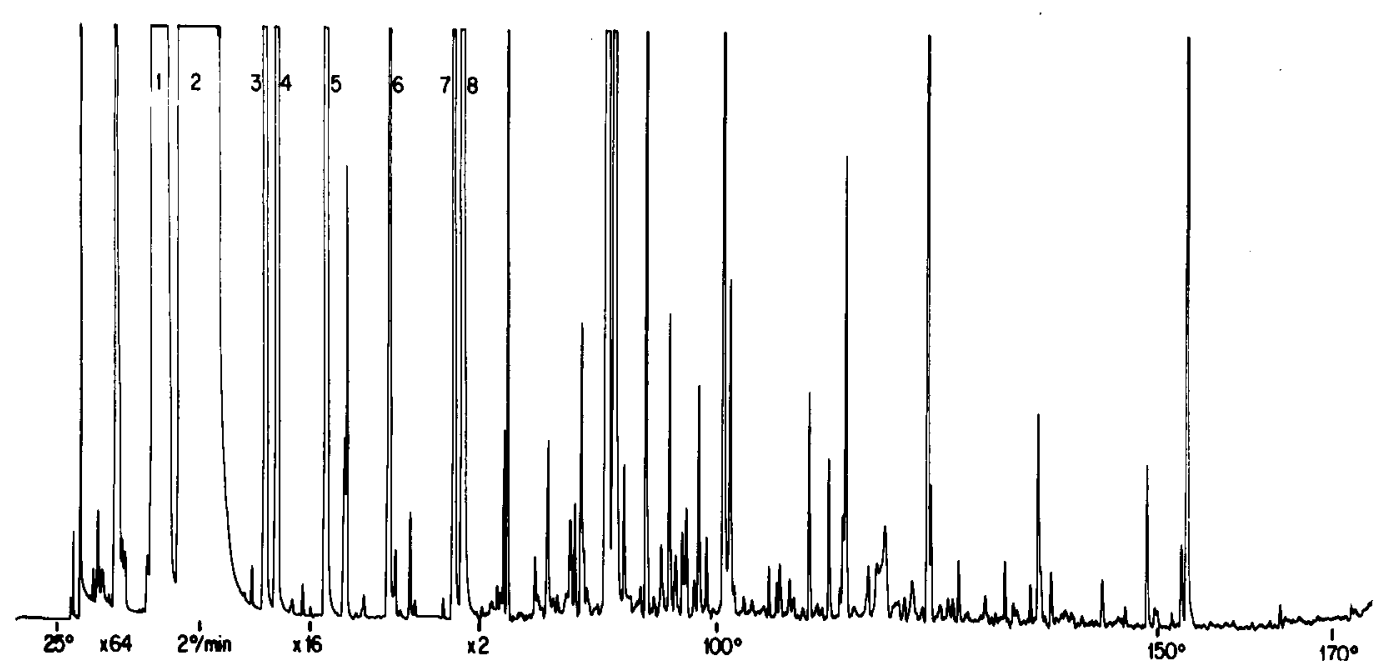

Fig. 4

- Analy sis of brandy. Column: $40 \mathrm{~m} \times 0.32 \mathrm{~mm}$, soft glass, leached with $\mathrm{HCl}$, treated with saturated Ba $(\mathrm{OH})_{2}$ at $25^{\circ} \mathrm{C}$, coated with Carbowax 400, film thickness $0.3 \mu \mathrm{m}$. Carrier hydrogen, $2.8 \mathrm{~cm}^{3} \mathrm{~min}^{-1}$. Injection: $1.5 \mathrm{~mm}^{3}$ at column temp. $25^{\circ}$, split ratio $1: 5$; effective sample size $0.3 \mathrm{~mm}^{3}$. Gas chromatograph Carlo Erba, Milano, Mod. 2100 , injector type 1976. Major components: 1 methanol, 2 ethanol, 3 2-butanol, 4 n-propanol, 5 2-methylpropanol, 6 n-butanol, 7 2-methyl-1-butanol, 8 3-methyl-1-butanol. To note: Enormous overloading in the alcohol section, without influence on the flavour section run with high sensitivity and stable base line up to $170^{\circ}$.

it on the glass surface $[2,4]$. However, random deposition of particles is likely to lack homogeneity as well as sufficient bonding to the support. These arguments prevented us from becoming active in this field.

\section{Practical Recommendations}

\subsection{Acidic Leaching}

The aim of acidic leaching is to produce a glass surface consisting of almost pure silica, which is the ideal support for barium carbonate crystallization. Thus, intense leaching reduces the surface differences between different glas types to almost zero.

Fill the raw glass capillary with $20 \% \mathrm{HCl}$, leaving empty $6-8 \%$ of total length. Seal both ends in a flame while they are connected to vacuum. During heating to $150^{\circ}$ the liquid will expand and will almost fill the empty space. We strongly recommend to use, at least for the first time, a glass door oven, allowing to check whether at $150^{\circ}$ there still remains a section of empty tubing, otherwise the capillary will break at once, as soon as it becomes filled! After keeping the capillary at $150^{\circ}$ over night, open the ends and introduce an amount of distilled water corresponding approx. to $20 \%$ of the internal volume, to flush out the acid. Heat the empty capillary to $300^{\circ}$ for several hours while applying a moderate flow of dry gas to it. Without this dehydrating treatment the silica surface would partly be destroyed by the barium hydroxide solution.

\subsection{Detergents for the Barium Carbonate Treatment}

The purpose of a detergent is to stabilize the water film remaining on the internal capillary wall behind the plug of barium hydroxide solution. This film must at least remain unbroken up to the end of the crystallization process, since, from a broken film, irregular deposits of barium carbonate would be formed. If crystallization at room temperature is applied the film must remain as long as carbon dioxide is fed to the capillary (for reason see chapter 2.3).

The nonylphenol polyethyleneoxide type detergent we recommended in our first report (e.g. Dehydrophen D) is suitable for crystallization at $80^{\circ}$. It is not sufficiently effective, however, for crystallization at room temperature. The results of our search for the ideal detergent may be summarized as follows. Anionogenic detergents failed since none of them remains soluble in the presence of barium ions. Cationogenic products also failed, since their common primary influence is rendering the glass surface hydrophobic. Thus nonionogenic types exclusively were further studied. The best results were observed with Marlazin L 10*), which is a dodecyl amino derivative of a carbowax containing 10 ethylene oxide moieties.

\subsection{Barium Hydroxide Solutions for Crystallization at Room Temperature}

The two essential components of the solution are barium hydroxide and detergent. For crystallization at $80^{\circ}$ from diluted solutions we previously recommended to add some $\mathrm{KOH}$ to speed up the uptake of carbon dioxide. For crystallization at room temperature we found this additive to be less helpful since it increases the amount of dissolved carbon dioxide in the surface film. Thus, for crystallization at room temperature we recommend to prepare the following solutions.

*) Manufactured by Chemische Werke Hüls AG, GFR; distributed by Dr. Bender and Dr. Hobein AG, Riedtlistr. 15, CH-8042 Zürich, Switzerland. 
Saturated barium hydroxide solution: Add approx. $50 \mathrm{~g}$ of $\mathrm{Ba}(\mathrm{OH})_{2} \cdot 8 \mathrm{H}_{2} \mathrm{O}$, reagent grade, to $500 \mathrm{~cm}^{3}$ of distilled water. Shake vigorously several times during half a day, and allow to settle over night.

Solution for polar coatings: Into a $50 \mathrm{~cm}^{3}$ flask weigh $5 \mathrm{mg}$ of Marlazin L 10, fill up with saturated barium hydroxide solution. Mix, and allow to stand over night. Solution for apolar coatings: Into a $50 \mathrm{~cm}^{3}$ flask weigh $1.5 \mathrm{mg}$ of Marlazin L 10. Add $45 \mathrm{~cm}^{3}$ of distilled and $\mathrm{CO}_{2}$-free water. After mixing add $1.5 \mathrm{~cm}^{3}$ of saturated barium hydroxide solution. Allow to stand over night. For both solutions we recommend a flask with screw cap and perforated internal plastic cap (Fig. 5). The internal cap remains in place when the end of a capillary is introduced into the solution. Thus, practically no atmospheric carbon dioxide enters the flask.

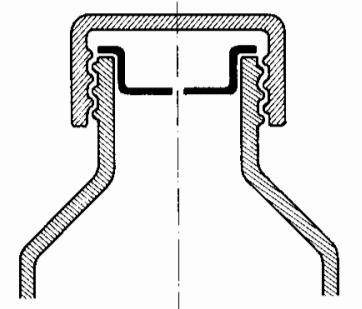

Fig. 5

- Closure of bottles containing barium hydroxide solution. Internal plastic cap with $1.5 \mathrm{~mm}$ bore for introducing the end of a glass capillary. We use the same closure for our gc-test solutions, to avoid evaporation of solvent.

\subsection{Barium Carbonate Treatment at Room Temperature}

Treatment for polar coatings: Push one end of a piece of $\mathrm{HCl}-$ leached glass capillary into a $0.5 \%$ solution of $\mathrm{HCl}$. By applying vacuum to the other end fill $5-10 \%$ of column length with the liquid. Repeat the procedure to fill $10-20 \%$ of column length ( $10 \%$ for $0.25 \mathrm{~mm}$ i.d., $20 \%$ for $0.4 \mathrm{~mm}$ i.d.) with saturated barium hydroxide solution containing $0.01 \%$ of Marlazin L 10 . The length of air plug between the liquid plugs is uncritical. Protect the column from draught and heat radiation. Adjust the length of the air plug (length of empty capillary at inlet end) to $5-12 \mathrm{~cm}(5 \mathrm{~cm}$ for $0.25 \mathrm{~mm}$ i.d., $8 \mathrm{~cm}$ for $0.3 \mathrm{~mm}$ i.d., $12 \mathrm{~cm}$ for $0.4 \mathrm{~mm}$ i.d.). Open the pressure control valve of the carbon dioxide supply to flush all dead volumes. Connect the column to the carbon dioxide supply and adjust the speed of the moving plugs to approx. $1 \mathrm{~cm} \mathrm{sec}^{-1}$. Connect a buffer capillary of similar inner diameter to the exit end of the column. Using a lens check the end of the alkaline liquid from time to time. No solid particulates must appear. They would be due to too short an air plug, allowing carbon dioxide to diffuse up to the plug end. They would lead to scattered large deposits. When all liquid has left the column replace the carbon dioxide by air or inert gas supply, adjusted to the same pressure as carbon dioxide. Keep the gas flowing for at least $2 \mathrm{~min}$ per $10 \mathrm{~m}$ of capillary length. After this step the crystals are sufficiently fastened to the glass surface that no more care is necessary to avoid temperature differences, touching or moving the column. Put the column in an oven and repeat, at $80-90^{\circ}$, flushing as carried out in the foregoing step (i.e. same gas flow and duration).

Remove the column. At room temperature push through a plug of acetone, the volume of which corresponds to $20 \%$ of column length, with a flow rate of approx. $1 \mathrm{~cm} \mathrm{sec}^{-1}$. Suggestions for manipulation and tools see Fig. 6. Meanwhile the buffer column has been rinsed by pushing back the liquids with a plug of acetone (whereby the acidic solution completely washes out the barium hydroxide). The dried buffer column is now connected to the exit of the column. When acetone has left the column, connect the exit to vacuum for at least the same duration as used for the two foregoing flushing steps. The column is now ready for polar coating.

Treatment for apolar coatings: The treatment includes the same steps and manipulations, with the following differences concerning materials and conditions. First liquid plug: $0.05 \% \mathrm{HCl}$, instead of $0.5 \%$. Barium hydroxide solution: 30 times diluted solution containing $0.003 \%$ of Marlazin L 10 (see 3.3). Length of air plug between barium hydroxide and carbon dioxide : $25 \mathrm{~cm}$ for $0.25 \mathrm{~mm}$ i.d., $40 \mathrm{~cm}$ for $0.3 \mathrm{~mm}$ i.d., $80 \mathrm{~cm}$ for $0.4 \mathrm{~mm}$ i.d. As a further difference, the first inactivation step may be combined with the drying step: After introducing acetone, immediately introduce the same plug length of $0.05 \%$ Carbowax $20 \mathrm{M}$ dissolved in methylene chloride. After drying with vacuum, mount the column in an oven, with inert gas flow as used for flushing. When the air in the column is completely replaced by inert gas, start heating. Keep the column at $280^{\circ}$ for 20 min under continuing flow. Cool the column slowly to avoid uptake of air into the open end. The final step, completing inactivation and improving the wettability, may be added immediately: Repeat rinsing and drying (same conditions as for acetone) with a $0.1 \%$ solution of Triton or Emulphor. Keep for $20 \mathrm{~min}$ at $240^{\circ}$, instead of $280^{\circ}$. The column is now ready for apolar coating.

\subsection{Preparation of Polar Columns}

Originally [1] we adapted the support surface to the polarity of a given liquid phase by treating the surface with a selected solution of barium hydroxide. According to our more recent experience column preparation can, without any qualitative disadvantage, be greatly simplified in the following way. For our purpose we term "polar" phases all phases which do not readily and permanently spread on smooth surfaces. For all these phases we use the same barium carbonate surface as obtained from the saturated barium hydroxide solution. On this surface the most polar phases such as Silar 10C, strongly polar polyesters, carbowaxes, are immediately deposited, with static or dynamic coating according to the viscosity of their solutions [1]. For less polar phases, such as OV-225, 


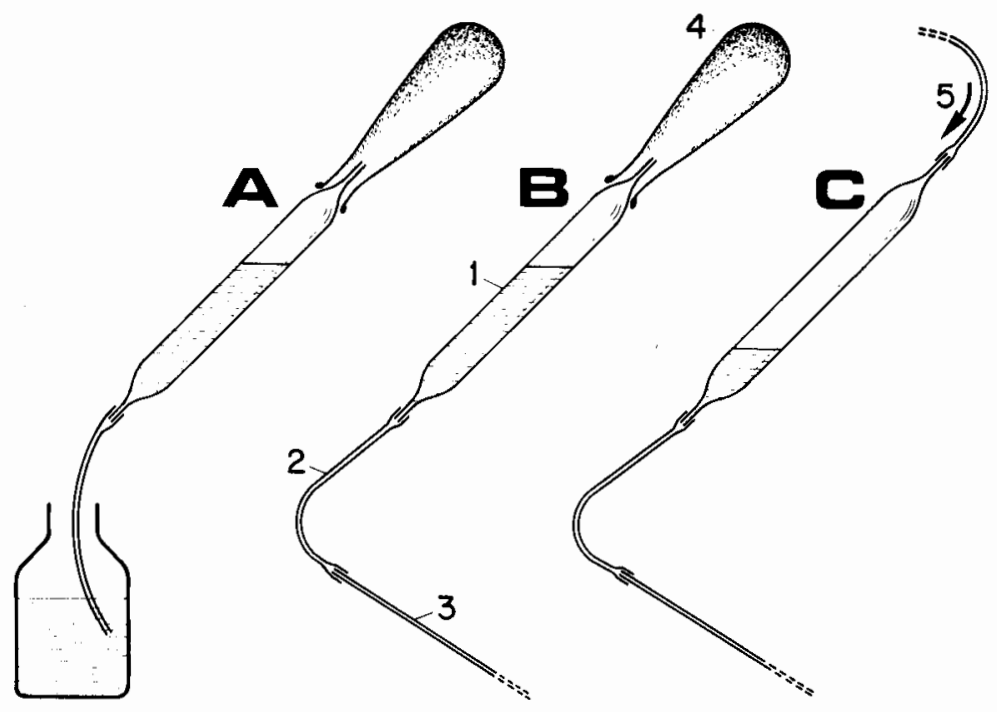

Fig. 6

- Tools and manipulation for introducing various liquids into a glass capillary column. 1 : glass tubing $6-10 \mathrm{~cm}$ long, $5 \mathrm{~mm}$ i.d., volume $1-2 \mathrm{~cm}^{3}$, with restricted ends. 2: PE or PTFE tubing, $0.5 \mathrm{~mm}$ i.d., ends widened with a hot needie. 3: end of capillary column. 4: pipette bulb, $1 \mathrm{~cm}^{3}$. 5: PE or PTFE tubing connected to pressure regulator of air or inert gas supply.
Emulphor, Ucon HB, we rinse the column with $0.05 \%$ carbowax $20 \mathrm{M}$ solution and heat to $280^{\circ}$ for $20 \mathrm{~min}$ (practical details see 3.4). For even less polar phases, such as $\mathrm{OV}-17$, Ucon LB, OS-124, we use the same carbowax treatment, followed by a treatment with $0.1 \%$ Ucon HB 5100 in methylene chloride, with heating to $240^{\circ}$ for $20 \mathrm{~min}$. The simple principle of these very rapid treatments is to treat the barium carbonate surface with a more polar phase than the phase intended for coating, and to avoid excessive polarity differences between these phases. Obviously the choice of phases for such treatments is very broad and opens extremely interesting opportunities to produce columns with specific adsorption/inactivation characteristics, whereby these characteristics are selected according to the special analytical application for which the column is prepared.

\subsection{Preparation of Apolar Columns}

The choice of phases yielding stable films on a smooth surface is rather limited. Besides pure alkanes and methyl silicones, it only includes phases like SE-52 and SE-54, which are very similar to the former ones. For this group of phases the barium carbonate treatment serves to cover the catalytically active silica surface, rather than to create a micro-roughness. Surface treatment follows again the principle as formulated in the foregoing chapter. A well tested procedure is the treatment first with carbowax, then with the bifunctional (i.e. containing a hydrophilic and a hydrophobic moiety) phase Triton or Emulphor, as indicated in chapter 3.4 .

It is a well known practise to add small amounts of polar phases to apolar ones to assure effective and longlasting inactivation. Unfortunately this simple means fails in our case. Even minute amounts (e.g. $0.01 \%$ of apolar phase) of Triton or Emulphor drastically reduce the life time of a column. It is essential, therefore, that remaining Carbowax or Emulphor, not permanently bound to the solid surface, be removed before coating. Since for apolar phases we use static coating exclusively, we proceed as follows.
Applying vacuum we fill one third of column length with chloroform. Then, without disconnecting vacuum, we switch from chloroform to the solution of the phase (e.g. $0.2 \%$ ) in methylene chloride. The air zone between the two liquids allows one to observe when the column becomes filled with the coating solution. The advantage of using chloroform as a precursor lies in its higher efficiency as a solvent for polar phases on one hand, and in its lower vapor pressure, facilitating work under vacuum, on the other hand. For more details see [1].

We greatly prefere gums (SE-52, SE-54, OV-1) before fluids. As main advantages we observe higher separation efficiencies and longer life time, while we observe almost no difference between gums and fluids concerning the lower temperature limit. We greatly regret that the actual choice of commercially available gum phases is so small.

\section{Acknowledgement}

This work has again generously been sponsored by F. J. Burrus \& Cie., Boncourt, Switzerland.

\section{Literature}

[1] K. Grob and G. Grob, J. Chromatog. 125, 471 (1976).

[2] E. Schulte, Chromatographia 9, 315 (1976).

[3] K. Grob, Chromatographia 7, 94 (1974).

[4] J. J. Franken, G. A. F. M. Rutten and J. A. Rijks, J. Chromatog. 126, 117 (1976).

[5] J. Krupcik, M. Kristin, M. Yalachovicoya and S. Janiga, J. Chromatog. 126, 147 (1976). 